\title{
Editorial
}

\section{The vital osteoclast: how is it regulated?}

\author{
D Aeschlimann", and BAJ Evans ${ }^{2}$ \\ ${ }^{1}$ Matrix Biology \& Tissue Repair Research Unit, Dental School, University of \\ Wales College of Medicine, Heath Park, Cardiff, CF14 4XY, UK \\ ${ }^{2}$ Department of Child Health, University of Wales College of Medicine, Heath \\ Park, Cardiff, CF14 4XY, UK \\ * Corresponding author: D Aeschlimann; E-mail: AeschlimannDP@Cardiff.ac.uk
}

Cell Death and Differentiation (2004) 11, S5-S7.

doi:10.1038/sj.cdd. 4401470

Bone is a rigid but dynamic organ. Once formed, it is continually broken down and reformed by the co-ordinated actions of osteoclasts (that mediate resorption) and osteoblasts (that mediate formation) on trabecular bone surfaces and in the Haversian systems of cortical bone. Any net change in bone mass therefore reflects a change in the balance between these two processes. If osteoclastic bone resorption exceeds the bone-forming capacity of osteoblasts, the result is osteoporosis, but if the opposite occurs the result is osteopetrosis. This remodelling occurs in focal and discrete packets - bone-remodelling units - throughout the skeleton. As the remodelling that occurs in each unit is geographically and chronologically separated from other units of remodelling, it is thought that activation of the sequence of cellular events responsible is locally controlled, probably by paracrine signalling in the bone microenvironment.

All diseases of bone are superimposed on this normal cellular remodelling process. Most are due to excess osteoclastic activity, which leads to an imbalance in bone remodelling that favours resorption. The past 5 years have witnessed important insights into osteoclast biology. Much of this new information has come from the analysis of a family of biologically related tumour necrosis factor (TNF) receptor (TNFR)/TNF-like proteins - osteoprotegerin (OPG), receptor activator of nuclear factor (NF) $-\kappa B$ (RANK) and RANK ligand (RANKL) - which together regulate osteoclast differentiation. ${ }^{1}$ Osteoclasts are derived from the monocyte/macrophage lineage, and proliferation and survival of their precursors depend on the cytokine macrophage colony-stimulating factor (M-CSF). Activation of RANK by osteoblast-expressed RANKL commits the precursors to the osteoclast fate. OPG is a soluble decoy receptor that competes with RANK for binding to RANKL. Studies of this pathway have clarified how diverse physiological and pathophysiological signals exert their effects on osteoclast formation, function and survival, and ultimately control skeletal remodelling and bone mass. ${ }^{2}$

Two papers ${ }^{3,4}$ in the current issue of Cell Death and Differentiation further extend our understanding of osteoclast formation and survival. The paper by Yang et al. ${ }^{3}$ proposes a new role for decoy receptor 3 (DcR3) in regulating osteoclastogenic differentiation of haematopoietic precursor cells, whereas the paper by Luegmayr et al. raises the possibility that osteoclast function and survival are regulated by exogenous cholesterol/lipoprotein levels. In the following, these findings are briefly discussed in their respective contexts.

In addition to M-CSF and RANKL, pro-inflammatory cytokines - TNF- $\alpha$ in particular - have been shown to have crucial roles in osteoclast differentiation. Oestrogen deficiency induces bone loss as a result of alterations in cytokine expression that either directly or indirectly modulate osteoclasts and their precursors, and thereby increase resorption. TNF receptor 1 (TNFR1)-deficient mice are not responsive to oestrogen depletion, and TNF- $\alpha$, acting through TNFR1, augments RANKL-induced osteoclastogenesis. ${ }^{5,6}$ It has also been shown that high concentrations of TNF- $\alpha$ promote in vitro osteoclast formation independently of RANKL, through NF- $\kappa \mathrm{B}$ and JNK activation. ${ }^{7}$ However, the complete lack of osteoclasts in both $R_{A N K}{ }^{-1-}$ mice and $R A N K L^{-1-}$ mice ${ }^{8,9}$ indicates that TNFR1 signalling alone is not sufficient in vivo. Nevertheless, a major role for TNF- $\alpha$ in inflammation-driven bone resorption - as seen in postmenopausal osteoporosis, rheumatoid arthritis, periodontitis and osteolytic malignancies - is demonstrated by the fact that wild-type $\mathrm{T}$ cells, but not TNF- $\alpha^{-1-} \mathrm{T}$ cells, restore oophorectomy-induced bone loss. ${ }^{5}$

The paper by Yang et al. ${ }^{3}$ demonstrates that decoy receptor 3 (DcR3) is a novel effector molecule that enhances osteoclastogenesis from monocytes/macrophages. DcR3 belongs to the TNFR superfamily and, similarly to OPG, exists as a soluble protein lacking the transmembrane domain. DcR3 interacts with several members of the TNF superfamily, including Fas ligand (FasL), LIGHT and TLA1, and might therefore have an immunomodulatory role by blocking interactions between these ligands and their respective receptors that are found on $T$ cells. However, recent studies suggest that DcR3 mediates signalling in cells of the monocyte/macrophage lineage via a mechanism that is distinct from its neutralizing effect on FasL, LIGHT and TLA1. Yang et al. ${ }^{3}$ show that DcR3 induces formation of active osteoclasts from monocytes/macrophages in a dose-dependent manner by specifically promoting TNF- $\alpha$ production other pro-inflammatory mediators are unaffected - and thereby inducing TNFR1-mediated signalling (Figure 1). The authors further show that DcR3 activates the ERK and p38 MAPK pathways (but not JNK) and that inhibitors of either pathway attenuate TNF- $\alpha$ production. This is in agreement with previous reports on the regulation of TNF- $\alpha$ expression in monocytes. Although this and a number of other studies provide clear evidence that DcR3 can act as an effector molecule and activate a number of signalling pathways in addition to its function as a decoy receptor, a cell-surface molecule that interacts with DcR3 remains to be identified.

Osteoporosis and cardiovascular disease are major health problems that increase in prevalence with age. They have traditionally been viewed as separate disease 


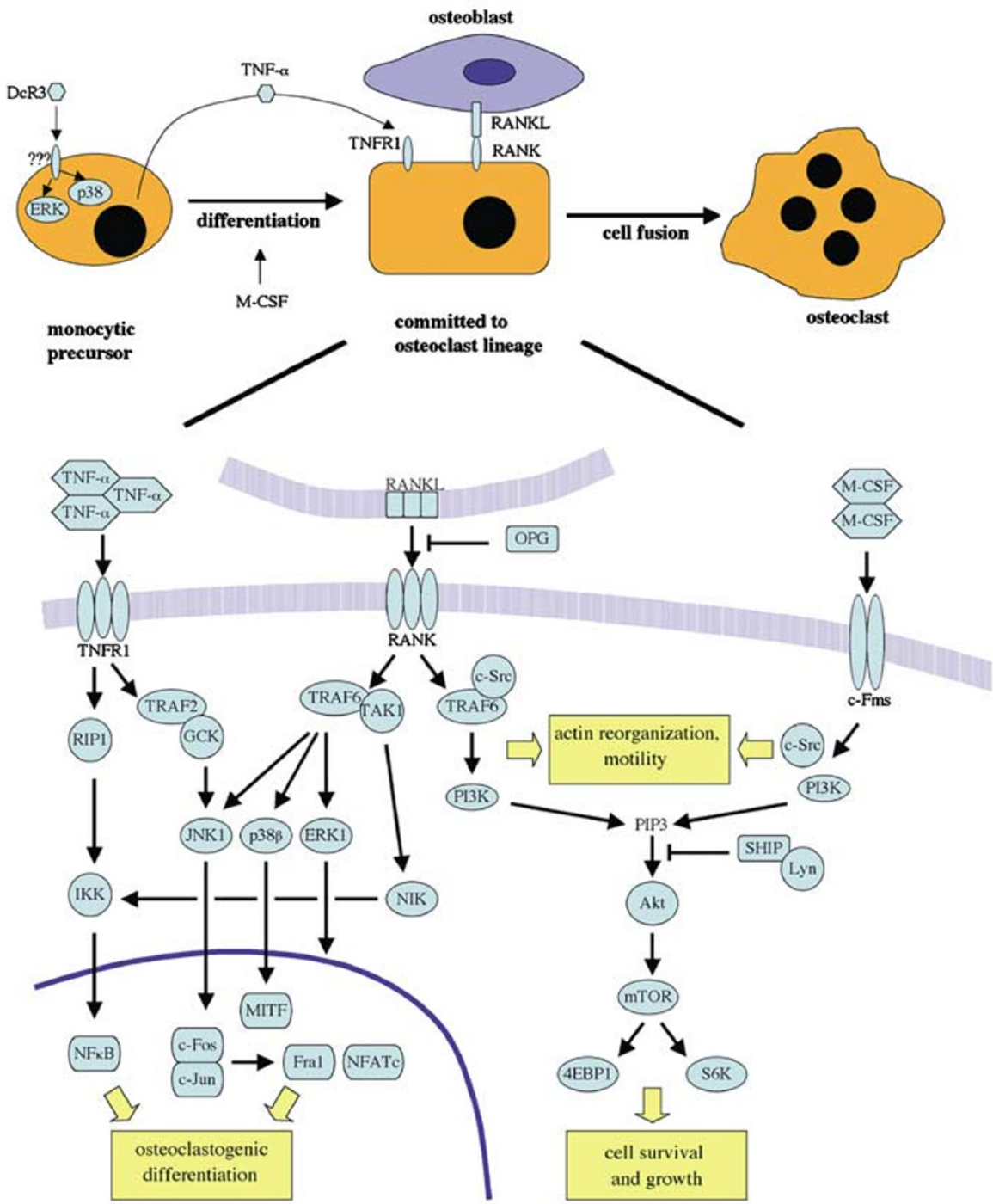

Figure 1 Osteoclastogenic differentiation. Binding of RANKL to RANK commits monocytic precursor cells to the osteoclastic lineage. Activation of RANK (which is prevented by OPG binding to RANKL) results in the recruitment of adaptor molecules, TNFR-associated factors (TRAFs) and activation of several downstream signalling pathways. RANKL-RANK complexes can activate all the three MAPK pathways, and the NF- $\kappa$ B pathway, by interacting with TRAF6 and TGF- $\beta$ activated kinase (TAK) 1. Activation of the JNK pathway mobilizes a number of osteoclastogenic transcription factors such as c-Fos, Fra-1 and nuclear factor-activated T-cells (NFAT) c1. Both the NF- $\kappa$ B and the JNK signalling pathway are essential for the differentiation of monocytic precursors into osteoclasts. Binding of TNF- $\alpha$ to TNFR1 has a strong synergistic effect with RANK signalling on the NF- $\kappa$ B and JNK pathways - through RIP1-IKK and TRAF2-GCK complex formation and activation, respectively. DcR3 induces TNF- $\alpha$ synthesis in mononuclear precursor cells by activating the ERK and p38 MAPK pathways via an interaction with an unknown cell-surface molecule. It thereby promotes osteoclastogenic differentiation. ${ }^{3}$ The RANKL-RANK-mediated interaction of TRAF6 with c-Src stimulates the PI3K-Akt pathway. This controls cytoskeletal changes in the osteoclast, as well as cell survival. M-CSF binding to c-Fms and its association with Src-family kinases and PI3K act synergistically on the PI3K-Akt pathway. Cholesterol depletion, by an unknown mechanism, affects Akt-mTOR signalling and induces osteoclast apoptosis ${ }^{4}$

entities, but accumulating evidence indicates that similar pathophysiological mechanisms underlie both diseases, and this has been recently reviewed by McFarlane et al. ${ }^{10}$ One possible link between the two diseases involves the cholesterol biosynthetic pathway, and some reports, ${ }^{11,12}$ but not all, ${ }^{13,14}$ have suggested that statin drugs used to treat hyperlipoproteinaemia may have a beneficial effect on bone mineral density. A recent study by Samuelson et al, ${ }^{15}$ however, indicates that cholesterol levels in women and men from young adulthood to middle age do not seem to affect the incidence of osteoporosis later in life.
M-CSF and RANKL - expressed by osteoblasts and preosteoblasts - promote proliferation and survival of osteoclasts and their precursors by activating the phosphatidylinositol-3 kinase (PI3K)-Akt pathway (Figure 1). Both c-Fms-deficient mice and mice lacking functional M-CSF (op/op mouse) have an osteoclast deficiency and associated osteopetrosis because of increased apoptosis of precursor cells, ${ }^{16}$ whereas mice deficient in SHIP-1 - a negative regulator of Akt that acts by dephosphorylating phosphatidylinositol-3,4,5-phosphate $\left(\mathrm{PIP}_{3}\right)$ - exhibit myeloid cell hyperplasia. ${ }^{17}$ Given that PI3K activity is critical for Akt activation and that c-Src stimulates 
PI3K in response to M-CSF and RANKL, C-Src may play a central role in monocyte and osteoclast survival, although its precise function remains controversial. ${ }^{18}$ Plasma membrane lipid rafts enriched in cholesterol, sphingolipids and a number of lipid-anchored proteins have recently been suggested to segregate signalling molecules and thereby to compartmentalize signal-transduction events within plasma membrane domains; however, their exact nature and function is only poorly understood. A recent study showed that disruption of lipid rafts in osteoclasts blocks RANKL-induced Akt activation, ${ }^{19}$ and as $\mathrm{c}$-Src is localized in lipid rafts it may be responsible for assembling antiapoptotic signalling complexes in membrane microdomains.

The paper by Luegmayr et al. ${ }^{4}$ provides evidence to suggest that osteoclast function and survival is highly dependent on exogenous sources of cholesterol. They show that cholesterol acceptors, including high-density lipoprotein, induce osteoclast apoptosis; that osteoclasts deficient in the low-density lipoprotein receptor (LDLR), in the presence of MCSF and RANKL, undergo apoptotic cell death at an increased rate compared with wild-type osteoclasts; and that osteoclasts are highly sensitive to forced cholesterol removal because of the absence of an endogenous cholesterol biosynthesis feedback response. Mechanistically, cholesterol depletion is suggested to promote apoptosis by affecting $\mathrm{M}$ CSF-stimulated Akt activation and the downstream mammalian target of rapamycin (mTOR)-ribosomal protein S6 kinase (S6K) pathways. Luegmayr et al. further show that osteoclasts from $\mathrm{LDLR}^{-1-}$ mice have cytoskeletal defects that affect morphology and possibly pre-osteoclast fusion. These are reversible by forced cholesterol uptake, and probably relate to the role of c-Src-PI3K in regulating cytoskeletal reorganization.

Most adult bone diseases relate to an imbalance in bone remodelling that is caused by increased osteoclast activity. Bone abnormalities caused by osteoclast gain of function are also associated with genetic conditions such as familial expansile osteolysis, expansile skeletal hyperphosphatasia and Paget's disease of bone. Understanding osteoclast regulation is therefore central to the development of new treatments for bone disorders. Low bone mass can be caused by either an increase in osteoclast number, or increased bone-resorptive capacity and prolonged survival of individual osteoclasts. The two papers in this issue of Cell Death and Differentiation provide new insights into both aspects, the survival and regulation of differentiation of this vital cell type.

\section{Key points}

- Apoptosis regulates osteoclast survival, and ultimately bone remodelling activity.

- Decoy receptor 3 induces TNF- $\alpha$ synthesis in monocytes/ macrophages via the p38 and ERK signalling pathways.

- High levels of TNF- $\alpha$ promote osteoclastogenic differentiation through TNFR1-mediated signal transduction.

- Cholesterol depletion inhibits the formation of large multinucleated osteoclasts and stimulates osteoclast apoptosis by reducing $\mathrm{M}-\mathrm{CSF}$ - and RANKL-mediated signalling through the Src-PI3K-Akt pathway.

\section{References}

1. Teitelbaum SL and Ross FP (2003) Nat. Genet. 4: 638-649

2. Boyle WJ et al. (2003) Nature 423: 337-342

3. Yang CR et al. (2004) Cell Death Differ. 11 (S1): S97-S107

4. Luegmayr E et al. (2004) Cell Death Differ. 11 (S2): S108-S118

5. Roggia C et al. (2001) Proc. Natl. Acad. Sci. USA 98: 13960-13965

6. Lam J et al. (2000) J. Clin. Invest. 106: 1481-1488

7. Kobayashi $\mathrm{K}$ et al. (2000) J. Exp. Med. 191: 275-286

8. Lacey DL et al. (1998) Cell 93: 165-176

9. Li J et al. (2000) Proc. Natl. Acad. Sci. USA 97: 1566-1571

10. McFarlane SI et al. (2004) Endocrine 23: 1-10

11. Edwards CJ et al. (2000) Lancet 355: 2218-2219

12. Watanabe $S$ et al. (2001) Am. J. Med. 110: 584-587

13. Reid IR et al. (2001) Lancet 357: 509-512

14. van Staa TP et al. (2001) JAMA 285: 1850-1855

15. Samelson EJ et al. (2004) Bone 34: 557-561

16. Dai XM et al. (2002) Blood 99: 111-120

17. Baran CP et al. (2003) J. Biol. Chem. 278: 38628-38636

18. Miyazaki T et al. (2004) J. Biol. Chem. 279: 17660-17666

19. Ha H et al. (2003) J. Biol. Chem. 278: 18573-18580 